PROCEEDINGS OF THE

AMERICAN MATHEMATICAL SOCIETY

Volume 129, Number 1, Pages 121-129

S 0002-9939(00)05477-0

Article electronically published on June 21, 2000

\title{
NORMALITY AND EXCEPTIONAL VALUES OF DERIVATIVES
}

\author{
WALTER BERGWEILER
}

(Communicated by Albert Baernstein II)

\begin{abstract}
We show that a family $\mathcal{F}$ of functions meromorphic in some domain is normal, if for all $f \in \mathcal{F}$ the derivative $f^{\prime}$ omits the value 1 and if the values that $f^{\prime}$ can take at the zeros of $f$ satisfy certain restrictions. As an application we obtain a new proof of a theorem of Langley which classifies the functions $f$ meromorphic in the plane such that $f$ and $f^{\prime \prime}$ have no zeros.
\end{abstract}

\section{INTRODUCTION AND RESULTS}

Hayman 9 proved in 1959 that if $f$ is meromorphic in the complex plane $\mathbb{C}$ and if $f(z) \neq 0$ and $f^{\prime}(z) \neq 1$ for all $z \in \mathbb{C}$, then $f$ is constant. The corresponding normality criterion is due to $\mathrm{Gu}$ 8]: the family of all functions $f$ meromorphic in a domain $D$ and having the property that $f(z) \neq 0$ and $f^{\prime}(z) \neq 1$ for all $z \in D$ is normal.

Here we shall generalize these results by allowing $f$ to have zeros, but restricting the values $f^{\prime}$ can take at the zeros of $f$.

Theorem 1. Let $D \subset \mathbb{C}$ be a domain and let $K$ and $\varepsilon$ be positive real numbers. Let $\mathcal{F}$ be the family of all functions $f$ meromorphic in $D$ which satisfy the following conditions:

(i) If $z \in D$, then $f^{\prime}(z) \neq 1$.

(ii) If $z \in D$ and $f(z)=0$, then $0<\left|f^{\prime}(z)\right| \leq K$.

(iii) If $\Delta$ is a disk in $D$ and if $f$ has $m \geq 2$ zeros $z_{1}, z_{2}, \ldots, z_{m} \in \Delta$, then there exists $k \in\{-1\} \cup\{1, \ldots, m-2\}$ such that

$$
\left|\sum_{j=1}^{m} f^{\prime}\left(z_{j}\right)^{k}-m^{k+1}\right| \geq \varepsilon .
$$

Then $\mathcal{F}$ is normal.

Theorem 2. Let $f$ be meromorphic in $\mathbb{C}$ and let $K$ and $\varepsilon$ be positive real numbers. Suppose that conditions (i)-(iii) of Theorem 1 are satisfied (with $D=\mathbb{C}$ ). Then $f$ has the form

$$
f(z)=\alpha z+\beta
$$

where $\alpha, \beta \in \mathbb{C}, \alpha \neq 1$, and $|\alpha| \leq K$.

Received by the editors January 5, 1999 and, in revised form, March 9, 1999.

1991 Mathematics Subject Classification. Primary 30D45, 30D30.

(C)2000 American Mathematical Society 
If $m=2$ in (iii), then the only possible choice for $k$ is $k=-1$, and (1) reduces to

$$
\left|\frac{1}{f^{\prime}\left(z_{1}\right)}+\frac{1}{f^{\prime}\left(z_{2}\right)}-1\right| \geq \varepsilon .
$$

The choice $k=0$ has been excluded in (iii) because (II) is never satisfied in this case.

For a function $f$ meromorphic in a domain $D$ we shall use the notation

$$
M_{f}:=f^{\prime}\left(f^{-1}(0)\right)=\left\{f^{\prime}(z): z \in D \text { and } f(z)=0\right\} .
$$

For $r>0$ and $a \in \mathbb{C}$ we put $D(a, r):=\{z \in \mathbb{C}:|z-a|<r\}$. With this terminology (ii) takes the form $M_{f} \subset \overline{D(0, K)} \backslash\{0\}$.

Choosing $k=-1$ we see that (iii) is satisfied for $\varepsilon=1$ if $M_{f} \subset\{z: \operatorname{Re} z<0\}$. More generally, if there exist $\delta>0$ and $p \in \mathbb{N}, p \geq 2$, such that

$$
M_{f} \subset\left\{z: \operatorname{Re} z<p+1-\delta \text { and }\left|z-\left(\frac{p}{2}+\delta\right)\right|>\frac{p}{2}+\delta\right\}
$$

then (iii) holds for some $\varepsilon>0$. This can be seen by choosing $k=-1$ for $m \leq p$ and $k=1$ for $m>p$.

The results of Hayman and $\mathrm{Gu}$ mentioned above correspond to the case that $M_{f}=\emptyset$. We note that the case of holomorphic functions is simpler; see $\S 4$.

As an application of Theorem 2, we give a new proof of the following result proved by Langley [1] in 1993.

Langley's Theorem. Let $f$ be meromorphic in $\mathbb{C}$ and suppose that $f$ and $f^{\prime \prime}$ do not have zeros. Then $f$ has the form $f(z)=e^{a z+b}$ or $f(z)=(a z+b)^{-n}$, where $a, b \in \mathbb{C}, a \neq 0$, and $n \in \mathbb{N}$.

This result had been conjectured by Hayman [9, p. 23]. Actually Hayman's conjecture dealt with the case that $f$ and $f^{(k)}$ do not have zeros, $k \geq 2$. The case $k \geq 3$ had been settled by Frank [6] in 1976 .

To deduce Langley's Theorem from Theorem 2, we define $h:=f / f^{\prime}$ and observe that the zeros of $h$ are the poles of $f$, and that $h^{\prime}(z)=-1 / n$ if $z$ is a pole of $f$ of multiplicity $n$. Thus $M_{h} \subset\{-1 / n: n \in \mathbb{N}\}$. Since $h^{\prime}=1-f^{\prime \prime} f /\left(f^{\prime}\right)^{2}$, we have $h^{\prime}(z) \neq 1$ for all $z \in \mathbb{C}$. Theorem 2 now implies that $h$ has the form $h(z)=\alpha z+\beta$, and this implies that $f$ has the form stated.

Applying this argument to Theorem 11 we obtain the following result, which may be considered as a normal family analogue of Langley's theorem.

Theorem 3. Let $D \subset \mathbb{C}$ be a domain and let $\mathcal{F}$ be the family of all functions $f$ meromorphic in $D$ such that $f$ and $f^{\prime \prime}$ do not have zeros. Then $\left\{f^{\prime} / f: f \in \mathcal{F}\right\}$ is normal.

For families of holomorphic functions this result is due to Schwick [20, Theorem 5.1]. More generally, Schwick shows that the conclusion holds for holomorphic families $\mathcal{F}$ if there exists $k \geq 2$ such that $f$ and $f^{(k)}$ have no zeros for all $f \in \mathcal{F}$.

To deduce Theorem 3 from Theorem 1, we proceed as above and note that if $f \in \mathcal{F}$, then $h:=f / f^{\prime}$ satisfies $M_{h} \subset\{-1 / n: n \in \mathbb{N}\}$ and $h^{\prime}(z) \neq 1$ for $z \in D$. The conclusion now follows from Theorem 1 . 


\section{Preliminary Lemmas}

We have already mentioned Hayman's result that if $f$ is meromorphic in $\mathbb{C}$ and non-constant, then $f$ takes the value 0 or $f^{\prime}$ takes the value 1 . For transcendental $f$, we have a stronger conclusion ([9, Theorem 3], see also [10, p. 60]).

Lemma 1. Let $f$ be transcendental and meromorphic in $\mathbb{C}$. Then $f$ or $f^{\prime}-1$ have infinitely many zeros.

The following result was proved in [3, Corollary 3].

Lemma 2. Let $f$ be meromorphic in $\mathbb{C}$ and of finite order. If $f$ has only finitely many critical values, then $f$ has only finitely many asymptotic values.

The following lemma is due to Rippon and Stallard [18, Lemma 2.2].

Lemma 3. Let $f$ be meromorphic in $\mathbb{C}$ and suppose that the set of all finite critical and asymptotic values of $f$ is bounded. Then there exists $R>0$ such that if $|z|>R$ and $|f(z)|>R$, then

$$
\left|f^{\prime}(z)\right| \geq \frac{|f(z)| \log |f(z)|}{16 \pi|z|} .
$$

This result is proved using a "logarithmic change of variable". For entire $f$ this device was used by Eremenko and Lyubich [5, § 2], and Lemma 3 can be deduced from their results in this case. The idea was carried over to meromorphic $f$ in [1, p. 173], and a version of Lemma 3 (with 16 replaced by a different constant) was stated explicitly in [2, Lemma 2]. However, the arguments used there rely on Lemma 8 in 1, and P. J. Rippon and G. M. Stallard have kindly pointed out to me that the proof of this lemma is false. In fact, the conclusion of [1 Lemma 8] is false, if the integer $p$ appearing there satisfies $p \geq 2$. (A counterexample for $p=2$, communicated to me by P. J. Rippon and G. M. Stallard, is given by $f(z)=\frac{\tan z}{z}+\frac{\pi}{2}$.) On the other hand, the conclusion of [1, Lemma 8] does hold if $p=1$. (This follows, for example, from [18, Lemma 2.1].) Since only the case $p=1$ is needed in [2], we see that Lemma 2 in [2] is correct.

The following result is due to Pang [16, Lemma 2]; see also [17, Lemma 2].

Lemma 4. Let $\mathcal{F}$ be a family of functions meromorphic in a domain D. Suppose that there exists $K>0$ such that $M_{g} \subset \overline{D(0, K)}$ for all $g \in \mathcal{F}$. If $\mathcal{F}$ is not normal, then there exist a sequence $\left(f_{n}\right)$ in $\mathcal{F}$, a sequence $\left(z_{n}\right)$ in $D$, a sequence $\left(\rho_{n}\right)$ of positive real numbers and a non-constant function $f$ which is meromorphic in $\mathbb{C}$ such that $z_{n} \rightarrow z_{0}$ for some $z_{0} \in D, \rho_{n} \rightarrow 0$ and

$$
\frac{f_{n}\left(z_{n}+\rho_{n} z\right)}{\rho_{n}} \rightarrow f(z)
$$

locally uniformly in $\mathbb{C}$. Moreover, the spherical derivative $f^{\#}$ of $f$ satisfies $f^{\#}(z) \leq$ $f^{\#}(0)=K+1$ for all $z \in \mathbb{C}$. In particular, $f$ has finite order.

In the conclusion of the lemma, it is possible to replace $f_{n}\left(z_{n}+\rho_{n} z\right) / \rho_{n}$ by $f_{n}\left(z_{n}+\rho_{n} z\right) / \rho_{n}^{\alpha}$, for any given $\alpha$ satisfying $-1<\alpha \leq 1$, but for our purposes the case $\alpha=1$ suffices. The papers cited above actually give a proof of this more general result. The case $\alpha=0$ is due to Zalcman [23], and the case $-1<\alpha<1$ is due to Pang [14, 15]. In this case no hypothesis on $M_{g}$ is required. For a discussion of this lemma and its applications we refer to a recent survey of Zalcman [24]. 


\section{Proof of Theorems 1 And 2}

We start with the following lemma. Generalizations of it will be discussed in $\S 4$.

Lemma 5. Let $f$ be meromorphic in $\mathbb{C}$ and of finite order. Suppose that $f^{\prime}(z) \neq 1$ for all $z \in \mathbb{C}$ and that there exists $K>0$ such that $M_{f} \subset \overline{D(0, K)}$. Then $f$ is rational and has the form

$$
f(z)=z+a+\frac{b}{(z+c)^{\ell}}
$$

with $a, b, c \in \mathbb{C}, b \neq 0, \ell \in \mathbb{N}$, or the form $f(z)=\alpha z+\beta$ with $\alpha, \beta \in \mathbb{C}, \alpha \neq 1$.

Proof. We consider the function $g(z)=z-f(z)$. Then $g^{\prime}(z)=1-f^{\prime}(z) \neq 0$ for all $z \in \mathbb{C}$.

First we assume that $f$ and hence $g$ are transcendental. By Lemma 1$] f$ has infinitely many zeros $z_{1}, z_{2}, \ldots$ We have $g\left(z_{k}\right)=z_{k}$ and $\left|g^{\prime}\left(z_{k}\right)\right|=\left|1-f^{\prime}\left(z_{k}\right)\right| \leq$ $1+K$ for all $k \in \mathbb{N}$. By Lemma 2 , $g$ has only finitely many asymptotic values, and thus satisfies the hypotheses of Lemma 3 for some $R>0$. We conclude that $\left|g^{\prime}\left(z_{k}\right)\right|>\left(\log \left|z_{k}\right|\right) / 16 \pi$ for large $k$, contradicting the estimate $\left|g^{\prime}\left(z_{k}\right)\right| \leq 1+K$ obtained for all $k$.

Thus $f$ and $g$ are rational. If $g$ is a polynomial, then $\operatorname{deg} g=1$ since $g^{\prime}$ does not have zeros, and it follows that $f$ has the form $f(z)=\alpha z+\beta$. If $g$ is not a polynomial, then we write $g=R+P / Q$ with polynomials $P, Q, R$ satisfying $\operatorname{deg} P<$ $\operatorname{deg} Q$. Since $g^{\prime}$ does not have zeros, we deduce that $R$ is constant. Thus $g^{\prime}=$ $\left(P^{\prime} Q-P Q^{\prime}\right) / Q^{2}$. Let $a_{1}, a_{2}, \ldots, a_{d}$ be the zeros of $P^{\prime} Q-P Q^{\prime}$, with multiplicities $m_{1}, m_{2}, \ldots, m_{d}$. Then each $a_{j}$ is a zero of $Q$ of multiplicity $m_{j}+1$. It follows that

$$
\operatorname{deg} P+\operatorname{deg} Q-1=\operatorname{deg}\left(P^{\prime} Q-P Q^{\prime}\right)=\sum_{j=1}^{d} m_{j}=\sum_{j=1}^{d}\left(m_{j}+1\right)-d \leq \operatorname{deg} Q-d .
$$

Hence $d=1$ and $\operatorname{deg} P=0$. This implies that $Q$ has the form $Q(z)=\alpha(z+\beta)^{\ell}$ with $\alpha, \beta \in \mathbb{C}, \alpha \neq 0, \ell \in \mathbb{N}$. It follows that $f$ has the form (2). This completes the proof of Lemma 5

We shall discuss functions of the form (2) in more detail. We denote the residue of a meromorphic function $F$ at a point $\xi$ by $\operatorname{res}(F, \xi)$.

Lemma 6. Let $f$ be a rational function of the form (2), with $a, b, c \in \mathbb{C}, b \neq 0$, $\ell \in \mathbb{N}$. Let $p \in\{0,1, \ldots, \ell\}$. Then

$$
\operatorname{res}\left(\frac{\left(f^{\prime}\right)^{p}}{f},-c\right)=1-(\ell+1)^{p} .
$$

Proof. The conclusion is clear for $p=0$ since $1 / f$ is holomorphic at $-c$ and thus $\operatorname{res}(1 / f,-c)=0=1-(\ell+1)^{0}$. For $p \geq 1$ we have

$$
f^{\prime}(z)^{p}=\left(1-\frac{b \ell}{(z+c)^{\ell+1}}\right)^{p}=\sum_{\nu=0}^{p}\left(\begin{array}{l}
p \\
\nu
\end{array}\right)(-b \ell)^{\nu}(z+c)^{-\nu \ell-\nu}
$$


and

$$
\begin{aligned}
\frac{1}{f(z)} & =\frac{b^{-1}(z+c)^{\ell}}{1+b^{-1}(z+c)^{\ell}(z+a)} \\
& =b^{-1}(z+c)^{\ell} \sum_{\mu=0}^{\infty}(-1)^{\mu} b^{-\mu}(z+c)^{\mu \ell}(z+a)^{\mu} \\
& =\sum_{\nu=1}^{\infty}(-1)^{\nu-1} b^{-\nu}(z+c)^{\nu \ell}(z+a)^{\nu-1} \\
& =\sum_{\nu=1}^{\infty}(-1)^{\nu-1} b^{-\nu} \sum_{j=0}^{\nu-1}\left(\begin{array}{c}
\nu-1 \\
j
\end{array}\right)(a-c)^{j-1-\nu}(z+c)^{\nu \ell+j}
\end{aligned}
$$

near $z=-c$. It follows that in the series for $1 / f$ only the terms with $j=\nu-1$ contribute to the residue of $\left(f^{\prime}\right)^{p} / f$ at $-c$. We thus obtain

$$
\operatorname{res}\left(\frac{\left(f^{\prime}\right)^{p}}{f},-c\right)=\sum_{\nu=1}^{p}\left(\begin{array}{l}
p \\
\nu
\end{array}\right)(-b \ell)^{\nu}(-1)^{\nu-1} b^{-\nu}=-\sum_{\nu=1}^{p}\left(\begin{array}{l}
p \\
\nu
\end{array}\right) \ell^{\nu}=1-(\ell+1)^{p} .
$$

Proof of Theorem 1. Suppose that $\mathcal{F}$ is not normal. We apply Lemma 4 to obtain sequences $\left(f_{n}\right),\left(z_{n}\right)$, and $\left(\rho_{n}\right)$ as there such that

$$
g_{n}(z):=\frac{f_{n}\left(z_{n}+\rho_{n} z\right)}{\rho_{n}} \rightarrow f(z)
$$

for some non-constant function $f$ which is meromorphic in $\mathbb{C}$ and of finite order. Moreover, $f^{\#}(z) \leq f^{\#}(0)=K+1$ for all $z \in \mathbb{C}$.

Let $\xi$ be a zero of $f$. Then $g_{n}$ has a zero $\xi_{n}$ for sufficiently large $n$ such that $\xi_{n} \rightarrow \xi$. Now $\zeta_{n}=z_{n}+\rho_{n} \xi_{n}$ is a zero of $f_{n}$, and since $f_{n}^{\prime}\left(\zeta_{n}\right)=g_{n}^{\prime}\left(\xi_{n}\right) \rightarrow f^{\prime}(\xi)$ we deduce from condition (ii) that $\left|f^{\prime}(\xi)\right| \leq K$. Thus $M_{f} \subset \overline{D(0, K)}$.

Suppose now that $f$ has the form $f(z)=\alpha z+\beta$ with $\alpha, \beta \in \mathbb{C}$. It follows that $|\alpha| \leq K$ and hence that

$$
f^{\#}(0)=\frac{|\alpha|}{1+|\beta|^{2}} \leq|\alpha|<K+1
$$

a contradiction. Thus $f$ does not have the form $f(z)=\alpha z+\beta$. In particular, $f^{\prime}(z) \not \equiv 1$. From condition (i) we can thus deduce that $f^{\prime}(z) \neq 1$ for all $z \in \mathbb{C}$.

Since we have already excluded the case that $f$ has the form $f(z)=\alpha z+\beta$, it now follows from Lemma 5 that $f$ has the form (2).

We put $m:=\ell+1$ and note that $f$ has $m$ zeros $\xi_{1}, \ldots, \xi_{m}$, counted according to multiplicity. Choose $R>\max _{1 \leq j \leq m}\left|\xi_{j}\right|$. For large $n$ there exist $m$ distinct zeros $\xi_{j, n} \in D(0, R)$ of $g_{n}$ such that $\xi_{j, n} \rightarrow \xi_{j}$ as $n \rightarrow \infty$ for $1 \leq j \leq m$. Then $\zeta_{j, n}:=z_{n}+\rho_{n} \xi_{j, n}$ is a zero of $f_{n}$ and $g_{n}^{\prime}\left(\xi_{j, n}\right)=f_{n}^{\prime}\left(\zeta_{j, n}\right)$ for $1 \leq j \leq m$. Moreover, $\zeta_{j, n} \in \Delta_{n}:=D\left(z_{n}, \rho_{n} R\right)$ for $1 \leq j \leq m$, and if $n$ is sufficiently large, then $\Delta_{n} \subset D$ and $f_{n}$ has no further zeros in $\Delta_{n}$.

For $k \in\{-1\} \cup\{1, \ldots, m-2\}$ we have

$$
\sum_{j=1}^{m} f_{n}^{\prime}\left(\zeta_{j, n}\right)^{k}=\sum_{j=1}^{m} g_{n}^{\prime}\left(\xi_{j, n}\right)^{k}=\sum_{j=1}^{m} \operatorname{res}\left(\frac{\left(g_{n}^{\prime}\right)^{k+1}}{g_{n}}, \xi_{j, n}\right) \rightarrow \sum_{\xi \in f^{-1}(0)} \operatorname{res}\left(\frac{\left(f^{\prime}\right)^{k+1}}{f}, \xi\right)
$$


as $n \rightarrow \infty$, where in the last sum multiple zeros $\xi$ of $f$ occur only once. Since

$$
\frac{f^{\prime}(z)^{k+1}}{f(z)}=\frac{1}{z}+O\left(\frac{1}{z^{2}}\right)
$$

as $z \rightarrow \infty$, the residue theorem and Lemma 6 imply that

$$
\sum_{j=1}^{m} f_{n}^{\prime}\left(\zeta_{j, n}\right)^{k} \rightarrow 1-\operatorname{res}\left(\frac{\left(f^{\prime}\right)^{k+1}}{f},-c\right)=(\ell+1)^{k+1}=m^{k+1}
$$

as $n \rightarrow \infty$. This contradicts condition (iii).

Proof of Theorem 2. Suppose that $f$ satisfies conditions (i)-(iii) of Theorem 1. Then, for each $\alpha \in \mathbb{C}$, the function $f_{\alpha}$ defined by $f_{\alpha}(z):=f(z+\alpha)$ also satisfies these conditions. By Theorem 1, the family $\left\{f_{\alpha}\right\}_{\alpha \in \mathbb{N}}$ is normal. By Marty's normality criterion, $f_{\alpha}^{\#}(0) \leq C$ for some $C>0$ and all $\alpha \in \mathbb{C}$. Thus $f^{\#}(z) \leq C$ for all $z \in \mathbb{C}$. Hence $f$ has finite order. Lemma 5 implies that $f$ has the form (2) or the form $f(z)=\alpha z+\beta$ with $\alpha, \beta \in \mathbb{C}$. Suppose that $f$ has the form (2). Using Lemma 6 we see as in the proof of Theorem 1 that if $m:=\ell+1$ and if $\xi_{1}, \ldots, \xi_{m}$ are the zeros of $f$ and $k \in\{-1\} \cup\{1, \ldots, m-2\}$, then

$$
\sum_{j=1}^{m} f^{\prime}\left(\xi_{j}\right)^{k}=1-\operatorname{res}\left(\frac{\left(f^{\prime}\right)^{k+1}}{f},-c\right)=m^{k+1} .
$$

This gives a contradiction to condition (iii).

\section{REMARKS}

1. It follows from the proof of Theorem 1 that if the poles of $f$ have multiplicity at least $p \geq 2$ for all $f \in \mathcal{F}$, then it suffices to assume that condition (iii) holds for all $m \geq p+1$. We also note that the condition $0 \notin M_{f}$ in (ii) is used only to allow $k=-1$ in (iii). We thus obtain the following result.

Theorem 4. Let $D \subset \mathbb{C}$ be a domain, $K>0, \varepsilon>0, p \in \mathbb{N}, p \geq 2$. Let $\mathcal{F}$ be the family of all functions $f$ meromorphic in $D$ which satisfy the following conditions:

(i) If $z \in D$, then $f^{\prime}(z) \neq 1$.

(ii) $M_{f} \subset \overline{D(0, K)}$.

(iii) All poles of $f$ have multiplicity at least $p$.

(iv) If $\Delta$ is a disk in $D$ and if $f$ has $m \geq p+1$ zeros $z_{1}, z_{2}, \ldots, z_{m} \in \Delta$, counted according to multiplicity, then there exists $k \in\{1, \ldots, m-2\}$ such that

$$
\left|\sum_{j=1}^{m} f^{\prime}\left(z_{j}\right)^{k}-m^{k+1}\right| \geq \varepsilon
$$

Then $\mathcal{F}$ is normal.

We note that (iv) is satisfied for some $\varepsilon>0$ if $M_{f} \subset\{z: \operatorname{Re} z<p+1-\delta\}$ for some $\delta>0$. Thus (iv) follows from (ii) if $K<p+1$.

We also mention that (ii) and (iv) are always satisfied if all $f \in \mathcal{F}$ have only multiple zeros, that is, if $M_{f}=\{0\}$. In this case the normality of $\mathcal{F}$ was proved 
by Wang and Fang [22, Theorem 5]. More generally, they show that $\mathcal{F}$ is normal if there exists $k \in \mathbb{N}$ such that each function $f \in \mathcal{F}$ has only zeros of multiplicity at least $k+1$ and poles of multiplicity at least 2 , and satisfies $f^{(k)}(z) \neq 1$ for all $z \in D$.

If all $f \in \mathcal{F}$ are holomorphic, then we can take $p$ in Theorem 4 arbitrarily large, and we are thus led to the following result.

Theorem 5. Let $D \subset \mathbb{C}$ be a domain and let $K>0$. Let $\mathcal{F}$ be the family of all functions $f$ holomorphic in $D$ which satisfy the following conditions:

(i) If $z \in D$, then $f^{\prime}(z) \neq 1$.

(ii) $M_{f} \subset \overline{D(0, K)}$.

Then $\mathcal{F}$ is normal.

The arguments used to deduce Theorem 2 from Theorem 1 now yield the following consequence of Theorem [5].

Theorem 6. Let $f$ be entire. If $f^{\prime}(z) \neq 1$ for all $z \in \mathbb{C}$ and if $M_{f}$ is bounded, then $f$ has the form $f(z)=\alpha z+\beta$ where $\alpha, \beta \in \mathbb{C}, \alpha \neq 1$.

Of course, Theorem 4 also has an analogue for functions meromorphic in the plane.

2. Condition (iii) cannot be omitted in Theorem 1. Similarly, condition (iv) is necessary in Theorem 4. This follows simply from the fact that for each $\ell \in \mathbb{N}$ the family of all functions $f$ of the form (2) is not normal. It does not seem unlikely, however, that we can omit condition (iii) in Theorem 2, provided we exclude functions of the form (21). Probably we can also allow $f$ to have multiple zeros.

Conjecture 1. Let $f$ be meromorphic in $\mathbb{C}$ and transcendental. If $f^{\prime}(z) \neq 1$ for all $z \in \mathbb{C}$, then $M_{f}$ is unbounded.

Lemma [5]says that the conjecture is true for functions of finite order. By Theorem 6 it also holds for entire functions.

Considering $g(z)=z-f(z)$ we see that Conjecture 1 is equivalent to the following one.

Conjecture 2. Let $g$ be meromorphic in $\mathbb{C}$ and transcendental. Suppose that $g^{\prime}$ does not have zeros. Then there exists a sequence $\left(z_{n}\right)$ of fixed points of $g$ such that $\left|g^{\prime}\left(z_{n}\right)\right| \rightarrow \infty$.

Langley and Zheng [13] have given lower bounds for the multipliers of the fixed points of a meromorphic function $g$ under the hypothesis that the set of critical and asymptotic values of $g$ is bounded. For example, they have shown [13, Theorem 3] that if $\sigma$ is less than the order of $g$, then there exists a sequence $\left(z_{n}\right)$ of fixed points of $g$ such that $\left|g^{\prime}\left(z_{n}\right)\right|>\left|z_{n}\right|^{\sigma / 2}$. In view of Lemma 2, their results apply in particular when $g$ has finite order and $g^{\prime}$ has no zeros. Thus their results also lead to a considerable improvement of Lemma 5 .

3. Langley ([11], see also [12]) considered not only the case that $f$ and $f^{\prime \prime}$ have no zeros, but the more general situation where $f$ and a second order linear differential polynomial in $f$ have no zeros. It is not clear to me whether the method employed here can be modified to yield such results. 
The result of Frank [6] dealing with the case that $f$ and $f^{(k)}$ have no zeros, $k \geq 3$, has also been generalized to the case that $f$ and a linear differential polynomial of order $k$ in $f$ do not have zeros. We refer to the work of Frank and Hellerstein [7], Steinmetz [21] and Brüggemann [4].

\section{ACKNOWLEDGMENT}

I would like to thank Jim Langley and Willi Schwick for helpful comments, and the referee for suggesting that I consider a normal family analogue of Langley's theorem.

\section{REFERENCES}

[1] W. Bergweiler, Iteration of meromorphic functions, Bull. Amer. Math. Soc., New Ser. 29 (1993), 151-188. MR 94c:30033

[2] - On the zeros of certain homogeneous differential polynomials, Arch. Math. 64 (1995), 199-202. MR 96a:30035

[3] W. Bergweiler and A. Eremenko, On the singularities of the inverse to a meromorphic function of finite order, Rev. Mat. Iberoam. 11 (1995), 355-373. MR 96h:30055

[4] F. Brüggemann, Proof of a conjecture of Frank and Langley concerning zeros of meromorphic functions and linear differential polynomials, Analysis 12 (1992), 5-30. MR 93e:30067

[5] A. Eremenko and M. Lyubich, Dynamical properties of some classes of entire functions, Ann. Inst. Fourier 42 (1992), 989-1020. MR 93k:30034

[6] G. Frank, Eine Vermutung von Hayman über Nullstellen meromorpher Funktionen, Math. Z. 149 (1976), 29-36. MR 54:10601

[7] G. Frank and S. Hellerstein, On the meromorphic solutions of nonhomogeneous linear differential equations with polynomial coefficients, Proc. London Math. Soc. III. Ser. 53 (1986), 407-428. MR 88k:30032

[8] Yongxing $\mathrm{Gu}$, On normal families of holomorphic functions (Chinese), Acta Math. Sinica 23 (1980), 157-161.

[9] W. K. Hayman, Picard values of meromorphic functions and their derivatives, Ann. Math., II. Ser. 70 (1959), 9-42. MR 22:1675

[10] — Meromorphic Functions, Clarendon Press, Oxford, 1964. MR 29:1337

[11] J. K. Langley, Proof of a conjecture of Hayman concerning $f$ and $f^{\prime \prime}$, J. London Math. Soc. II. Ser. 48 (1993), 500-514. MR 94k:30075

[12] _ On second order linear differential polynomials, Result. Math. 26 (1994), 51-82. MR 95k:30059

[13] J. K. Langley and J. H. Zheng, On the fixpoints, multipliers and value distribution of certain classes of meromorphic functions, Ann. Acad. Sci. Fenn., Math. 23 (1998), 133-150. MR 99b:30044

[14] Xuecheng Pang, Bloch's principle and normal criterion, Sci. China, Ser. A 32 (1989), 782-791. MR 91i:30031

[15] _ On normal criterion of meromorphic functions, Sci. China, Ser. A 33 (1990), 521-527. MR 92b:30041

[16] $\_$, Shared values and normal families, preprint.

[17] Xuecheng Pang and L. Zalcman, Normal families and shared values, Bull. London Math. Soc., to appear.

[18] P. J. Rippon and G. M. Stallard, Iteration of a class of hyperbolic meromorphic functions, Proc. Amer. Math. Soc. to appear. CMP 98:09

[19] J. Schiff, Normal Families, Springer, New York, Berlin, Heidelberg, 1993. MR 94f:30046

[20] W. Schwick, Normality criteria for families of meromorphic functions, J. Analyse Math. 52 (1989), 241-289. MR 90k:30061

[21] N. Steinmetz, On the zeros of $\left(f^{(p)}+a_{p-1} f^{p-1}+\cdots+a_{0} f\right) f$, Analysis 7 (1987), 375-389. MR 89e:34059

[22] Yuefei Wang and Mingliang Fang, Picard values and normal families of meromorphic functions with multiple zeros, Acta Math. Sin., New Ser. 14 (1998), 17-26. 
[23] L. Zalcman, A heuristic principle in complex function theory, Amer. Math. Monthly 82 (1975), 813-817. MR 52:757

[24] - Normal families: new perspectives, Bull. Amer. Math. Soc., New Ser. 35 (1998), 215-230. MR 99g:30048

Mathematisches Seminar, Christian-Albrechts-Universität Zu Kiel, Ludewig-MeynStr. 4, D-24098 Kiel, Germany

E-mail address: bergweiler@math.uni-kiel.de 